\title{
ARTICLE
}

Translational Therapeutics

\section{M2 macrophages mediate sorafenib resistance by secreting HGF in a feed-forward manner in hepatocellular carcinoma}

\author{
Ningning Dong ${ }^{1}$, Xiangyi Shi ${ }^{1}$, Suihai Wang ${ }^{1}$, Yanjun Gao ${ }^{1}$, Zhenzhan Kuang ${ }^{1}$, Qian Xie ${ }^{1}$, Yonglong Li ${ }^{1}$, Huan Deng ${ }^{1}$, Yingsong Wu ${ }^{1}$, \\ Ming $\mathrm{Li}^{1}$ and Ji-Liang $\mathrm{Li} i^{1,2,3}$
}

BACKGROUND: Sorafenib is the only approved first line systemic therapy for advanced hepatocellular carcinoma (HCC) in the last decade. Tumour resistance to sorafenib has been of major obstacles to improve HCC patient survival.

METHODS: We polarised THP-1 cells to M1 and M2 macrophages, performed various in vitro assays and developed sorafenibresistant xenograft models to investigate the role of tumour-associated macrophages (TAM)-secreted molecules in HCC resistance to the targeted therapy.

RESULTS: We demonstrated M2, but not M1, macrophages not only promote proliferation, colony formation and migration of hepatoma cells but also significantly confer tumour resistance to sorafenib via sustaining tumour growth and metastasis by secreting hepatocyte growth factor (HGF). HGF activates HGF/c-Met, ERK1/2/MAPK and PI3K/AKT pathways in tumour cells. Tumourassociated M2 macrophages were accumulated in sorafenib-resistance tumours more than in sorafenib-sensitive tumours in vivo and produced abundant HGF. HGF chemoattracts more macrophages migrated from surrounding area, regulates the distribution of M2 macrophages and increases hepatoma resistance to sorafenib in a feed-forward manner.

CONCLUSIONS: Our results provide new insights into the mechanisms of sorafenib resistance in HCC and rationale for developing new trials by combining sorafenib with a potent HGF inhibitor such as cabozantinib to improve the first line systemic therapeutic efficacy.

British Journal of Cancer (2019) 121:22-33; https://doi.org/10.1038/s41416-019-0482-x

\section{BACKGROUND}

Hepatocellular carcinoma (HCC) is the sixth most commonly diagnosed cancer and the fourth leading cause of cancer-related death worldwide, ${ }^{1}$ characterised by rapid progression with high post-operation recurrence and high metastasis. ${ }^{2}$ Currently, standardised treatments of HCC patients include surgical resection, liver transplantation, transcatheter arterial chemoembolization, local radiofrequency ablation, and systemic targeted therapy with sorafenib or lenvatinib in the first-line ${ }^{3-5}$ and regorafenib ${ }^{6,7}$ or nivolumab in the second-line setting after progression on sorafenib. ${ }^{8,9}$ Although early-stage of or localised HCC are curable by surgical resection, liver transplantation or local ablation, $80 \%$ of HCC patients are diagnosed at advanced disease stages when only systemic therapy with sorafenib followed by regorafenib or nivolumab shows to improve patient survival. ${ }^{2}$

Sorafenib, is a small-molecule inhibitor of up to 40 kinases, potently inhibiting proangiogenic receptor tyrosine kinases including VEGFR-1/2/3, PDGFR- $\beta$, and FGFR1, and other kinases involved in tumorigenesis (Raf-1, wild type B-Raf, mutant B-Raf, CKit, Flt-3, and RET). ${ }^{10,11}$ Preclinical studies have demonstrated sorafenib effectively inhibited tumour growth of various cancer types. ${ }^{10}$ In 2008, the SHARP phase III trial showed that sorafenib substantially increased median survival in patients with advanced stage of HCC from 7.9 to 10.7 months. ${ }^{5}$ The beneficial effect of sorafenib was validated in another independent Sorafenib-AP phase III trial that showed an extension of median survival from 4.2 to 6.5 months. ${ }^{3}$ As a result, sorafenib has become the standard of care for treatment of advanced HCC since 2007. However, due to intra-nodule and inter-nodule tumour heterogeneity and heterogeneity in tumour evolution, ${ }^{12}$ the response rate to sorafenib is very low and the effective duration is short in clinical trials, ${ }^{3,5,13}$ suggesting intrinsic primary and acquired secondary resistance. Indeed, tumour resistance to sorafenib has become a major obstacle to the effectiveness of systemic therapy against HCC since then. Thus, understanding of the resistance mechanisms and identification of molecular markers to stratify the patients for sorafenib therapy will improve the clinical benefits by developing new therapeutic approaches or rational drug combinations. $^{14}$

Collective evidence shows that most studies on sorafenib resistance in HCC have been focused on tumour cells. Various mechanisms are involved in hepatoma resistance to sorafenib, including epithelial-mesenchymal transition (EMT) of tumour cells, ${ }^{15}$ cancer stem cells (CSC) or tumour-initiating cells, ${ }^{16,17}$

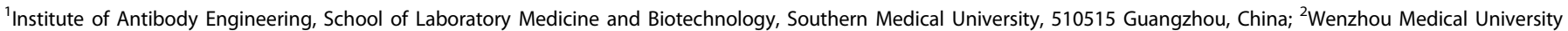

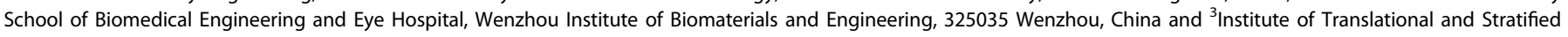
Medicine, University of Plymouth Faculty of Medicine and Dentistry, Plymouth PL6 8BU, UK

Correspondence: Yingsong Wu (wg@smu.edu.cn) or Ming Li (mingli2006_2006@126.com) or Ji-Liang Li (Ji-liang.li@plymouth.ac.uk)

These authors contributed equally: Ningning Dong, Xiangyi Shi

Received: 21 November 2018 Revised: 6 March 2019 Accepted: 2 May 2019

Published online: 27 May 2019 
activation of numerous growth factor pathways such as AR/EGFR pathway $^{18}$ and PI3K/AKT pathway, ${ }^{19,20}$ c-Jun activation, ${ }^{21}$ hypoxia, ${ }^{22}$ cancer cell metabolism, ${ }^{23}$ and autophagy, ${ }^{24}$ among others. $^{21}$ However, growing evidence has also uncovered the importance of stroma cells in tumour microenvironment (TME) in HCC progression ${ }^{25}$ and response to sorafenib by cross-talking with tumour cells. ${ }^{26}$ These may include tumour-associated endothelia, ${ }^{22}$ tumour-associated neutrophils, ${ }^{27}$ cancer-associated fibroblasts, ${ }^{28,29}$ tumour-infiltrated lymphocytes such as NK cells ${ }^{30}$ and myeloid cells, ${ }^{28}$ and tumour-associated macrophages (TAM). ${ }^{31-33}$

We are interested in hepatocarcinogenesis and its potential translation for development of either novel targeted therapies or predictive markers for therapeutic efficacy and/or patient prognosis. $^{34}$ In this paper, we report the role of M2-type of TAMs in hepatoma resistance to sorafenib by secreting hepatocyte growth factor (HGF). HGF activates HGF/c-Met, MAPK/ERK1/2, and PI3K/ AKT pathways in tumour cells, further recruits M2 TAMs, and thus sustains hepatoma growth and metastasis in a feed-forward manner.

\section{METHODS}

Cell lines and culture

Human acute monocytic leukaemia cell line THP-1 and hepatoma cancer cell lines (SMMC-7721, Hep3B, and Sk-hep1) were purchased from and authenticated by the Typical Culture Preservation Commission Cell Bank, Chinese Academy of Sciences (Shanghai, China). THP-1 cells were maintained in RPMI 1640 medium (Gibco BRL, New York, USA) while all hepatoma cells were cultured in DMEM (Gibco BRL, New York, USA) supplemented with $10 \%$ foetal bovine serum (FBS) (Gibco BRL, New York, USA) (complete medium) at $37^{\circ} \mathrm{C}$ with $5 \% \mathrm{CO}_{2}$.

Polarisation of M1-like and M2-like macrophages

Production and polarisation of THP-1-derived macrophages were conducted as described previously ${ }^{35,36}$ with some modifications. Briefly, $1 \times 10^{7}$ THP- 1 cells in $10-\mathrm{cm}$ dish were incubated in $10 \mathrm{ml}$ complete medium containing $200 \mathrm{ng} / \mathrm{ml}$ PMA (Sigma) for $24 \mathrm{~h}$ and cultured in $10 \mathrm{ml}$ fresh complete medium for another $24 \mathrm{~h}$ to produce THP-1 macrophages (M0). For M1 polarisation, the macrophages were then cultured in $10 \mathrm{ml}$ fresh complete medium with $100 \mathrm{ng} / \mathrm{ml}$ LPS (Sigma) and $20 \mathrm{ng} / \mathrm{ml} \mathrm{IFN-} \gamma$ (Sigma) for another $24 \mathrm{~h}$; whereas for M2 polarisation the macrophages were cultured $10 \mathrm{ml}$ fresh complete medium with $20 \mathrm{ng} / \mathrm{ml} \mathrm{IL-4}$ (Peprotech) and $20 \mathrm{ng} / \mathrm{ml} \mathrm{IL-13} \mathrm{(Peprotech)} \mathrm{for} 72 \mathrm{~h}$. After polarisation, $10^{6} \mathrm{M} 1$-like and $\mathrm{M} 2$-like macrophages were cultured in $10 \mathrm{ml}$ serum-free RPMI 1640 medium for $48 \mathrm{~h}$, respectively. The conditioned media (CM) were then harvested by centrifugation at $1300 \mathrm{rpm}$ for $5 \mathrm{~min}$ and the supernatant was aliquoted and stored at $-80^{\circ} \mathrm{C}$ for further use.

Flow cytometry analysis

After the polarisation, THP-1-derived macrophages were harvested and washed in PBS. $1 \times 10^{6}$ cells were then stained using antiCD11b-PE-Cy7, CD209-FITC, CD163-PE, CD115-PE-Cy7, CD204-FITC, CD206-APC (BD Pharmingen, USA) for $30 \mathrm{~min}$ at $4{ }^{\circ} \mathrm{C} .1 \times 10^{6}$ cells isolated from fresh xenograft tissues were stained using FITC-antimouse F4/80 (Bio-Rad), FITC-anti-mouse CD206 (Biolegend). An isotype-matched IgG was used as negative control. Results were analysed by flow cytometry (FACSCalibur, BD).

RNA extraction and real-time quantitative PCR (RT-qPCR) Total RNAs were extracted using Trizol reagent (Invitrogen) and converted into cDNA using the PrimeScript ${ }^{\mathrm{TM}}$ RT Master Mix (Takara). SYBR ${ }^{\oplus}$ Premix Ex Taq $^{\text {TM }}$ (Takara) was then used for RT-qPCR with the Applied Biosystems 7500 software. GAPDH was used as an internal control. Relative gene expression at the mRNA level was calculated using $2^{-\Delta C T}\left(\Delta C T=C t^{\text {target gene }}-\mathrm{CT}^{\mathrm{GAPDH}}\right)$.

Primer sequences for various genes are: HLA-DR forward (5'ATCATGACAAAGCGCTCCAACTAT- $3^{\prime}$ ), reverse (5'-GATGCCCACCAG ACCCACAG-3'); MRC1 forward (5'-GGCGGTGACCTCACAAGTAT-3'), reverse (5'-TITTCATGGCTTGGTTCTCC-3'); HGF forward (5'-CGAGG CCATGGTGCTATACT-3'), reverse (5'-GCATTCAGTTGTTCCAAAGG$\left.3^{\prime}\right)$; TNF-a forward (5'-GGCTCCAGGCGGTGCTTG-3'), reverse (5'-CA GATAGATGGGCTCATACCA-3'); CXCL-9 forward (5'-GGACTATCCAC CTACAATCCTTG- $\left.3^{\prime}\right)$, reverse $\left(5^{\prime}\right.$ - TITTAATCAGTTCCTTCACATCTGC3'; IL-10 forward (5'-GACTITAAGGGTTACCTGGGTTG-3'), reverse (5'-TCACATGCGCCTTGATGTCTG-3'); IGF-1 forward (5'-GCTCTTCAG TTCGTGTGTGGA-3'), reverse (5'-GCCTCCTTAGATCACAGCTCC-3');

VEGF forward (5'-CCTTGCTGCTCTACCTCCAC-3'), reverse $\left(5^{\prime}\right.$-GCA GTAGCTGCGCTGATAGA-3'); TGF- $\beta$ forward ( $5^{\prime}$-CCCAGCATCTGCA AAGCTC-3'), reverse ( $5^{\prime}$-GTCAATGTACAGCTGCCGCA-3'); IL-12 forward (5'-CATCAGGGACATCATCAA-3'), reverse (5'-GTCAGGGA GAAGTAGGAA-3'); and GAPDH forward (5'-CCACCCATGGCAAA TTCC-3'), reverse (5'-TGGGATTTCCATTGATGACAA-3').

Enzyme-linked immunosorbent assays (ELISA)

HGF in CM was measured by Human HGF ELISA Kit (Neobioscience, USA) on the Bio-Rad microplate reader (USA) following the manufacturer's instructions.

Cell proliferation assays

Total $1 \times 10^{4}$ tumour cells (SMMC-7721, Hep3B or Sk-hep1) per well were in triplicate seeded in 24-well plates for $24 \mathrm{~h}$ and then incubated with the CM, $3 \mu \mathrm{M}$ sorafenib (Sigma) $(10 \mathrm{mM}$ stock solution prepared with DMSO), $50 \mathrm{ng} / \mathrm{ml}$ recombinant human HGF $(\mathrm{R} \& D)$, or $200 \mathrm{ng} / \mathrm{ml}$ anti-HGF neutralising antibody (Abcam) for another 48 h. Twenty microlitre Cell Counting Kit-8 (CCK-8; Sigma, USA) solution was then added to each well. After incubated at $37^{\circ}$ $\mathrm{C}$ for $4 \mathrm{~h}$, the absorbance at $450 \mathrm{~nm}$ was measured on the Bio-Rad microplate reader. The experiment was repeated at least three times.

\section{Colony formation assays}

Colony formation assays were performed with 6-well plates (500 or 1000 cells/well) as described previously. ${ }^{34}$ Briefly, an equal amount of different treatment cells was plated in 6-well plates triplicate. After incubated at $37^{\circ} \mathrm{C}$ with $5 \% \mathrm{CO}_{2}$ for $15-20$ days, during which the supernatant in each well was replaced with fresh complete medium every 2-3 days, cell colonies formed were stained with $0.5 \%$ crystal violet following the fixation by methanol for counting. The experiment was repeated at least three times.

\section{Boyden chamber migration assays}

To determine HCC cell migration affected by M1-like or M2-like THP-1-derived macrophages, tumour cells were seeded in the top of insert well in CM or serum-free RPMI 1640 medium (Corning), while medium containing $10 \%$ FBS was placed in the lower chamber. After incubation at $37{ }^{\circ} \mathrm{C}$ with $5 \% \mathrm{CO}_{2}$, tumour cells migrated through the pores of insert well toward the chemoattractant below were stained in $0.5 \%$ crystal violet solution for $10 \mathrm{~min}$ and washed by running water for $10 \mathrm{~min}$. Images were captured under microscope and the number of migrated cells were counted. The experiment was repeated at least three times.

Western blotting

Western blotting was performed as described previously. ${ }^{34}$ Primary antibodies include rabbit anti-MET, pMET, ERK1/2, pERK1/2 (Thr202/Tyr204), AKT, and pAKT (Cell Signalling, USA). Primary rabbit anti-GAPDH antibody was used as a protein loading control. All assays were repeated at least three times.

Xenograft models

Female BALB/C nude mice aged 4-6 weeks old were purchased from the Experimental Animal Centre at Southern Medical 
University and maintained under standard pathogen-free conditions. All experimental procedures were approved by the Ethical Committee of Southern Medical University and animal welfares were closely monitored in accordance with the Guide for the Care and Use of Laboratory Animals of the National Institutes of Health. Tumour xenografts were essentially conducted as described previously. ${ }^{34}$ Each group contained 5-7 mice. SMMC-7721 tumour cells $\left(10^{6}\right.$ in $\left.100 \mu \mathrm{l}\right)$ were subcutaneously implanted into each mouse. Tumour volume was calculated with the formula of [length $\times$ width $\left.^{2} \times(\pi / 6)\right]$. When tumours reached $100 \mathrm{~mm}^{3}$ volume, mice were treated with sorafenib daily $(30 \mathrm{mg} / \mathrm{kg})$ via oral gavage. ${ }^{37}$ Mice were killed, and tumour cells were isolated from both sorafenib sensitive and resistant tumours as described in isolation of tumour tissue cells. Tumour cells isolated from the first round of xenograft tissues were cultured for $~ 48-h$ in vitro. Equal amount of tumour cells adhered on culture dishes from two mice (having similar tumour sizes) of either DMSO group or sorafenib group were then mixed (50:50\% ratio) in each group. The mixed tumour cells were then for the second round of xenograft growth by subcutaneous injection of nude mice as potential sorafenibsensitive (SS) and sorafenib-resistant (SR) tumours respectively. Sorafenib treatments were started when the second round of tumours reached $100 \mathrm{~mm}^{3}$. Mice were killed when tumour reached to $800 \mathrm{~mm}^{3}$. Tumours were then collected for either tissue CD31 staining or cell isolation for flow cytometry analysis of $\mathrm{F} 4 / 80^{+}$and $\mathrm{CD}^{206^{+}}$expressions.

Isolation of tumour tissue cells

Fresh xenograft tumours were soaked in PBS and chopped to $1 \mathrm{~mm}^{3}$ size with surgical blade. After washed with PBS, the slices were digested with collagenase (type IV, $1.5 \mathrm{mg} / \mathrm{ml}$, Sigma) and hyaluronidase (type $\mathrm{l}, 0.25 \mathrm{mg} / \mathrm{ml}$, Sigma) DMEM culture medium by rotating at $37^{\circ} \mathrm{C}$ for $2 \mathrm{~h}$. Digested cells were filtered through a sterilised cell strainer $(70 \mu \mathrm{m})$ and collected by centrifugation at $800 \mathrm{rpm} / \mathrm{min}$ for $5 \mathrm{~min}$. After washed in $25 \mathrm{ml} \mathrm{PBS}$, the isolated cells were either cultured for $\sim 48-h$ for the second round of xenograft growth or resuspended in $5 \mathrm{ml}$ RBC lysis buffer for $10 \mathrm{~min}$. After washed in PBS, the cells were then aliquoted in $10^{6}$ cells $/ 100 \mu$ l culture medium for flow cytometry analysis of $\mathrm{F} 4 / 80^{+}$and $\mathrm{CD}^{206^{+}}$expressions.

\section{Statistics}

Data were presented as mean \pm standard error of the mean (SEM). The SPSS 20.0 software (SPSS Inc., USA) and Prism 6 software were used for data analysis. The analysis of variance (ANOVA) test was used to compare mean values among three or more groups whereas independent-sample two-sided Student's $t$-test was used to compare two groups with normal distribution data. Statistical significance was indicated by an asterisk $\left({ }^{*} P<0.05,{ }^{*} P<0.01\right.$, ${ }^{* * *} P<0.001$, and $\left.{ }^{* * *} P<0.0001\right)$.

\section{RESULTS}

Polarisation of THP-1 to M1-like and M2-like macrophages

To investigate the effect of macrophage-secreting proteins on innate resistance of HCC to sorafenib, we induced the differentiation of THP-1 cells with PMA to produce macrophages (M0) and then polarised the macrophages with LPS/IFN- $\gamma$ and IL-4/IL-13, respectively. ${ }^{35,36}$ THP-1-derived M0 macrophages showed grapelike shapes while M1-like macrophages developed bipolar morphology in contrast to M2-like macrophages with clear bipolar extensions (Fig. 1a). Flow cytometry analysis demonstrated that $\mathrm{CD} 11 \mathrm{~b}$ was equally expressed in both $\mathrm{M} 1$-like and M2-like macrophages while the expression of CD163, CD115/CSF-1R, CD204/MSR1/SR-A, CD206/MRC1, or CD209/DC-SIGN was significantly higher in M2-like cells than that of M1-like cells (Fig. 1b). RTqPCR analysis confirmed that M1 markers such as HLA-DR, CXCL9, and TNFa were significantly upregulated in M1-like macrophages polarised with LPS and IFN- $\gamma$ whereas M2 marker, CD206/MRC1, was dramatically increased in THP-1-derived macrophages treated with IL-4 and IL-13 (Fig. 1c). Functional assays showed the abilities of proliferation and colony formation of either SMMC-7721 or Hep3B were significantly increased when incubated with $\mathrm{CM}$ from M2-like macrophages (M2-CM) compared to that of M1-like macrophages (M1-CM) (Fig. 1d, e). Together, the results suggested the polarised M1-like and M2-like THP-1 cells have intrinsic traits of tumour-associated M1 and M2 macrophages, respectively.

M2 macrophages confer hepatoma resistance to sorafenib by secreting molecules

We first investigated the inhibitory efficacy of sorafenib against tumour cells growing in serum-free medium. We showed that IC50 of sorafenib against SMMC-7721, Hep3B, and Sk-hep1 was 1.729 $\mu \mathrm{M}, 1.248 \mu \mathrm{M}$, and $3.421 \mu \mathrm{M}$, respectively (Fig. 2a). We then treated tumour cells with sorafenib at $3 \mu \mathrm{M}$ in the presence of $\mathrm{CM}$ harvested from the macrophages cultured at $48 \mathrm{~h}$. The ability of cell proliferation (Fig. 2b) or colony formation (Fig. 2c) was significantly inhibited by sorafenib for both SMMC-7721 and Hep3B cells compared to the vehicle control (Sor versus DMSO, $P<0.001)$. However, tumour cells became highly resistant to sorafenib in the presence M2-CM $(M 2+$ Sor versus Sor, $P<0.001)$. In contrast, M1-CM showed no protection to tumour cells (M1+Sor versus Sor, $P>0.05$ ). In addition, tumour migration was also significantly inhibited by sorafenib (Sor versus DMSO, $P<0.001$ ) (Fig. 2d). Again, tumour migration was significantly increased by M2-CM (M2+Sor versus Sor, $P<0.001)$ but not by M1-CM $(M 1+$ Sor versus Sor, $P>0.05)$. Thus, the results suggested that $M 2$ macrophages confer hepatoma resistance to sorafenib via sustaining tumour growth and metastasis by secreted soluble molecules.

HGF mediates sorafenib resistance conferred by M2 macrophages To investigate which molecules secreted from M2 macrophages are responsible for the sorafenib resistance, we screened a variety of gene expressions differentially between $M 1$ and $M 2$ macrophages at mRNA levels by RT-qPCR (Fig. S1). Of them, HGF expression was greatly upregulated in $M 2$ macrophages compared to that of M1 macrophages (Fig. 3a, left panel). ELISA revealed that the amount of HGF protein in CM secreted by $M 2$ macrophages was 30 -folds more than that of $M 1$ macrophages (Fig. 3a, right panel). We then investigated if exogenous HGF confers HCC resistance to sorafenib by performing proliferation and colony formation assays through incubation of tumour cells with $50 \mathrm{ng} / \mathrm{ml}$ recombinant human HGF. Exogenous HGF significantly increased the growth and colony formation of tumour cells in the presence of sorafenib (Fig. 3b, c, HGF+Sor versus Sor, $P<0.001)$. Also, exogenous HGF remarkably increased HCC migration (Fig. $3 \mathrm{~d}$ ) and significantly abolished the inhibitory effect of sorafenib on the migration of SMMC-7721 and Hep3B cells (Fig. 3e).

To investigate if HGF produced by $\mathrm{M} 2$ macrophages directly confers HCC resistance to sorafenib, we added anti-HGF antibody $(200 \mathrm{ng} / \mathrm{ml})$ to neutralise the HGF activity in M2-CM. As shown in Fig. 4, M2-CM increased cell proliferation, colony formation and migration of both SMMC-7721 and Hep3B (M2+Sor versus Sor, all $P<0.01)$. However, addition of antiHGF antibody abolished the proliferation (Fig. 4a), colony formation (Fig. 4b), and migration (Fig. 4c) of both SMMC-7721 and Hep3B cells promoted by M2-CM (M2+Sor+aHGF versus M2 + Sor, all $P<0.01$ ), suggesting a dominant role of HGF in sorafenib resistance conferred by $\mathrm{M} 2-\mathrm{CM}$.

M2 macrophages confer sorafenib resistance by activation of ERK/ MAPK and PI3K/AKT pathways through HGF/c-Met signalling To explore the possible mechanisms by which $M 2$ macrophages induce sorafenib resistance, we investigated the expression and 

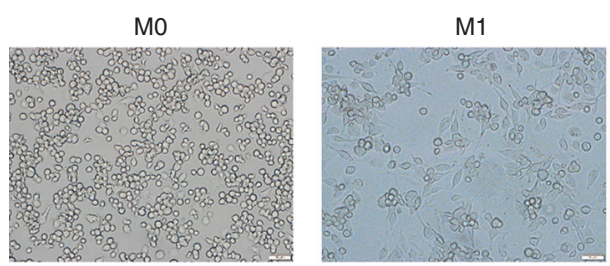

b
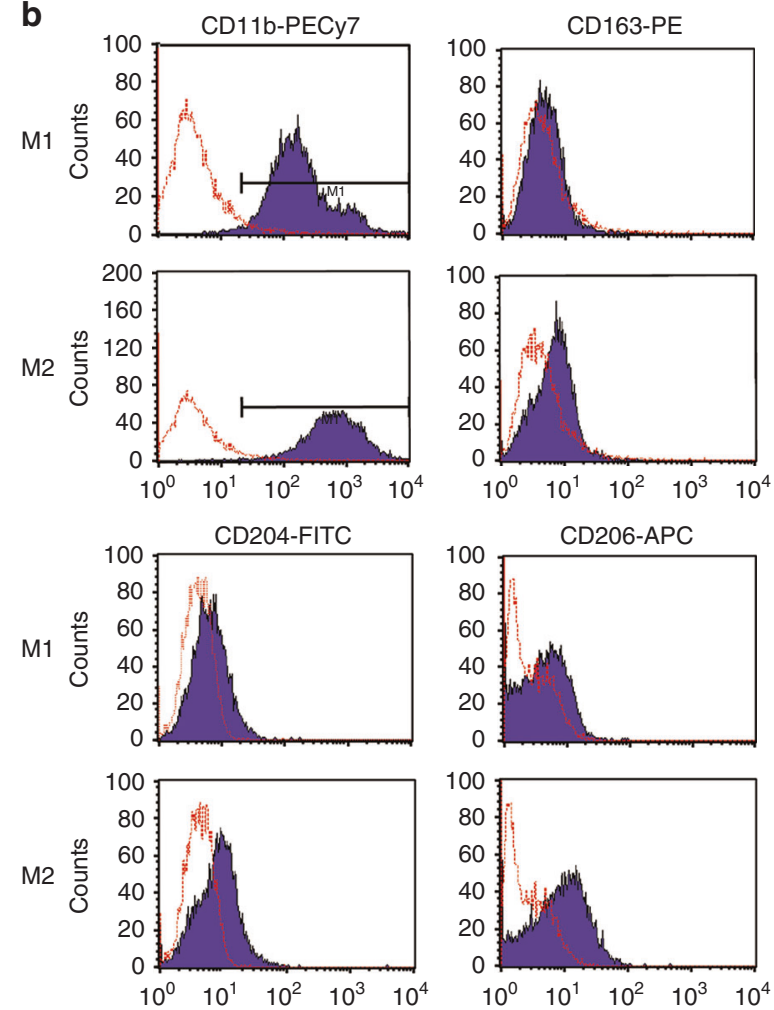

C
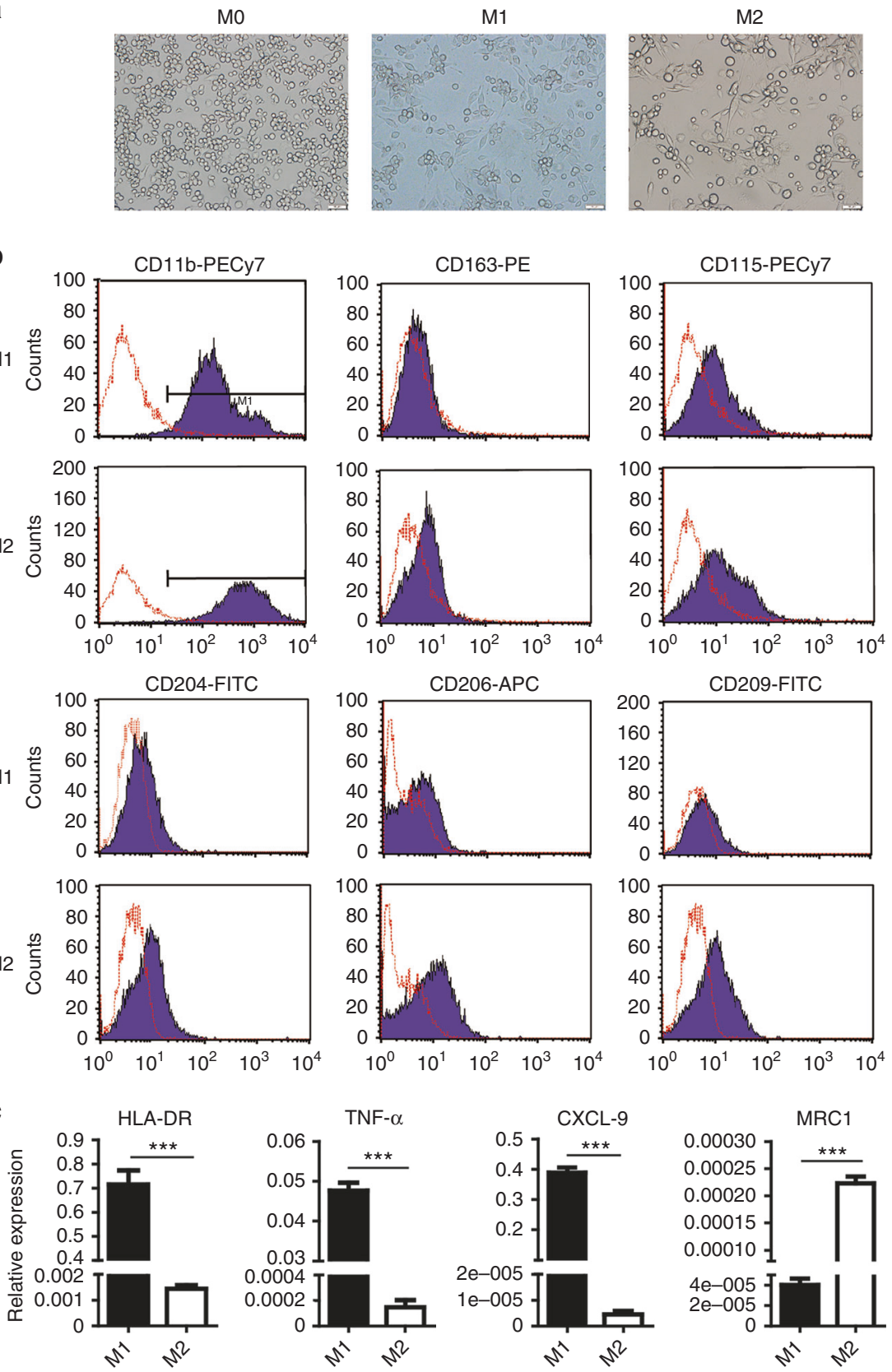
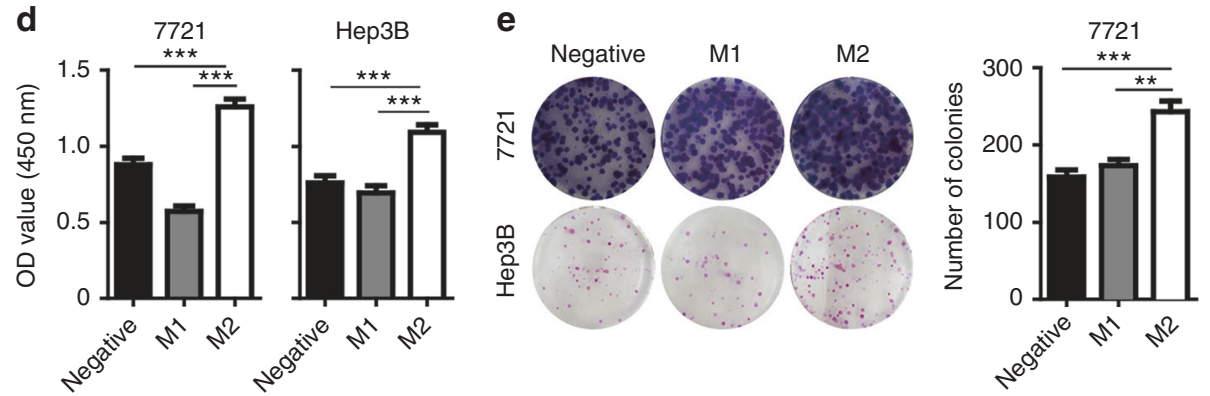

Fig. 1 Polarisation of THP-1 to M1-like and M2-like macrophages. a Cell morphology of M0-like, M1-like, or M2-like macrophages.

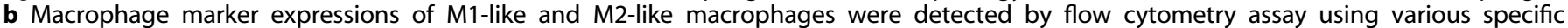
antibody (black histograms) or isotype-matched antibody (red histogram). c Expressions of HLA-DR, TNF- $\alpha$, CXCL-9 and MRC1 in M1 or M2 macrophages was determined by RT-qPCR. d Effects of M1-CM and M2-CM on the proliferation (at 48 h) of SMMC-7721 (left) and Hep3B cells

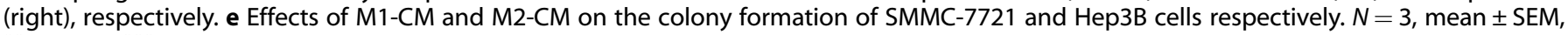
$* * P<0.01, * * * P<0.001$ 

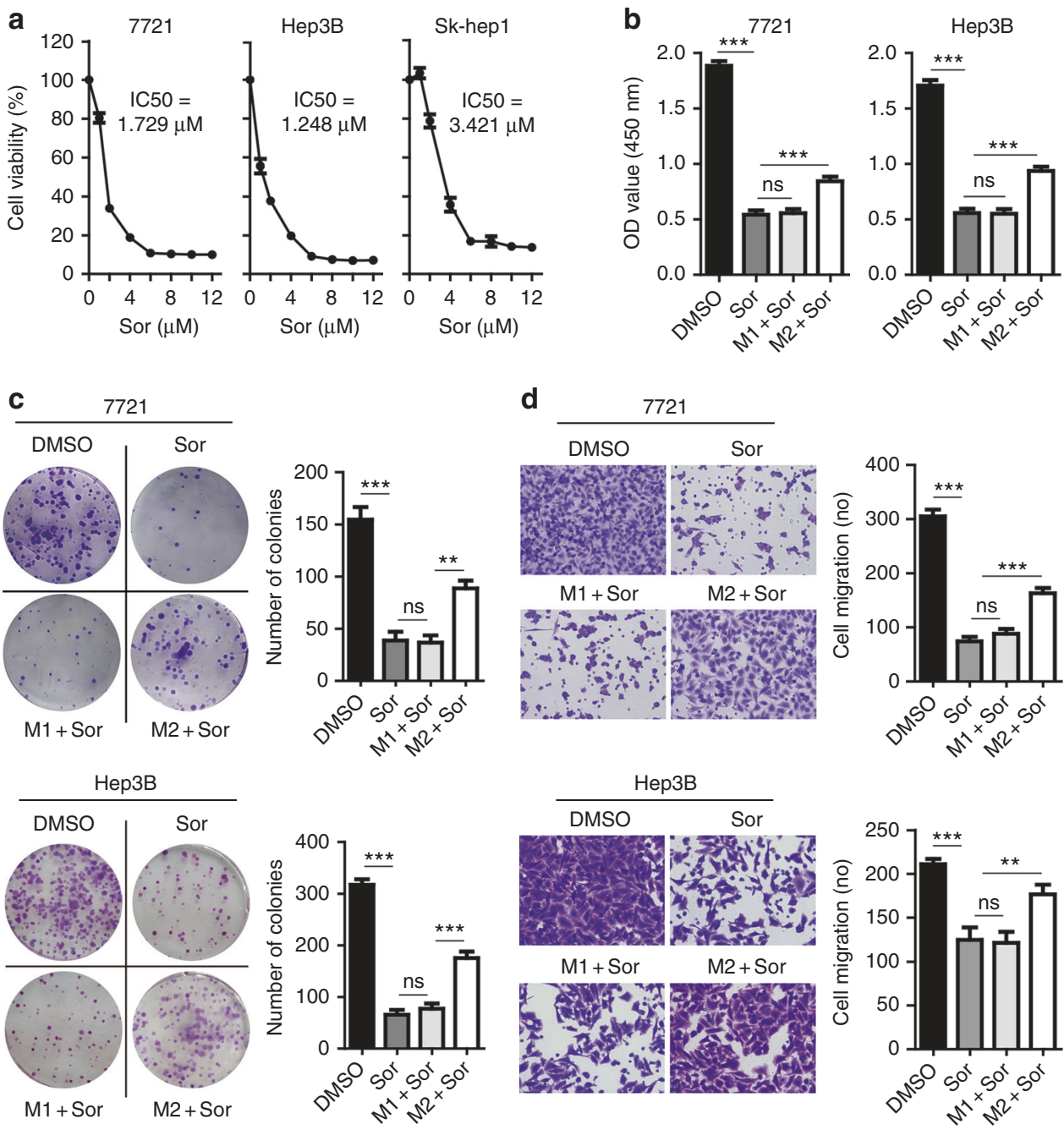

Fig. 2 M2 macrophages confer hepatoma resistance to sorafenib by secreting molecules. a Sorafenib inhibited the growth of SMMC-7721, Hep3B, and Sk-hep1 cells cultured in serum-free medium for $48 \mathrm{~h}$. IC50 of sorafenib against SMMC-7721, Hep3B, and Sk-hep1 is $1.729 \mu \mathrm{M}$, $1.248 \mu \mathrm{M}$, and $3.421 \mu \mathrm{M}$, respectively. b CCK-8 assays showing M2-CM but not M1-CM attenuated the ability of sorafenib (Sor) to inhibit the proliferation of SMMC-7721 or Hep3B cells. c Colony formation assays showing M2-CM but not M1-CM attenuated the ability of sorafenib to inhibit colony formation of SMMC-7721 or Hep3B cells. d Boyden chamber transwell assays showing M2-CM but not M1-CM attenuated the ability of sorafenib to inhibit cell migration of SMMC-7721 or Hep3B cells. $N=3$, mean \pm SEM, ${ }^{* *} P<0.01,{ }^{* * *} P<0.001, N S$, no significance

phosphorylation of key components in HGF/c-Met, ERK/MAPK, and PI3K/AKT pathways in tumour cells of SMMC-7721 and Hep3B from different treatment groups. Western blot analysis showed that sorafenib treatment did not significantly affect the expression and phosphorylation of c-Met (Fig. 5a, b). However, incubation of tumour cells with either M2-CM or exogenous HGF significantly increased the expression and phosphorylation of c-Met. Neutralising HGF by addition of anti-HGF antibody eliminated the increment of c-Met expression and phosphorylation enhanced by $\mathrm{M} 2-\mathrm{CM}$ or recombinant HGF, suggesting a dominant role of $\mathrm{HGF}$ in $\mathrm{M} 2-\mathrm{CM}$ in the sorafenib resistance.

Sorafenib did not affect the expression of ERK1/2 but dramatically reduced the phosphorylation level of both pERK1/2, confirming the role of sorafenib in inhibition of ERK1/2/MAPK pathway by targeting RAF. ${ }^{10}$ Incubation of tumour cells with either M2-CM or exogenous HGF partially abolished the suppression of pERK1/2 phosphorylation by sorafenib. However, neutralising HGF in M2-CM by anti-HGF antibody sustained the function of sorafenib, supporting the role of HGF in M2-CM in induction of
pERK1/2 phosphorylation. Similar results were obtained for the expression and phosphorylation of AKT, suggesting that the effect of M2-CM on ERK/MAPK and PI3K/AKT signalling is only partially mediated by HGF/c-Met signalling.

Accumulation of tumour-associated macrophages in sorafenibresistant tumour

To investigate if HGF regulates recruitment of TAMs in tumour microenvironment, we initially performed Boyden chamber migration assays. M2 macrophages showed strong intrinsic migratory ability. Exogenous HGF significantly chemoattracted the migration of $M 2$ macrophages but not $M 1$ macrophages (Fig. 6a), suggesting that HGF secreted by M2-like TAMs may recruit more macrophages in tumour tissues, regulate the distribution of M2 macrophages and increase tumour resistance to sorafenib in a feed-forward manner.

We then developed SS and SR tumour models by passaging SMMC-7721 cells in immunocompromised mice for two rounds (Fig. 6b). ${ }^{16}$ Tumour cells isolated from the first round of 

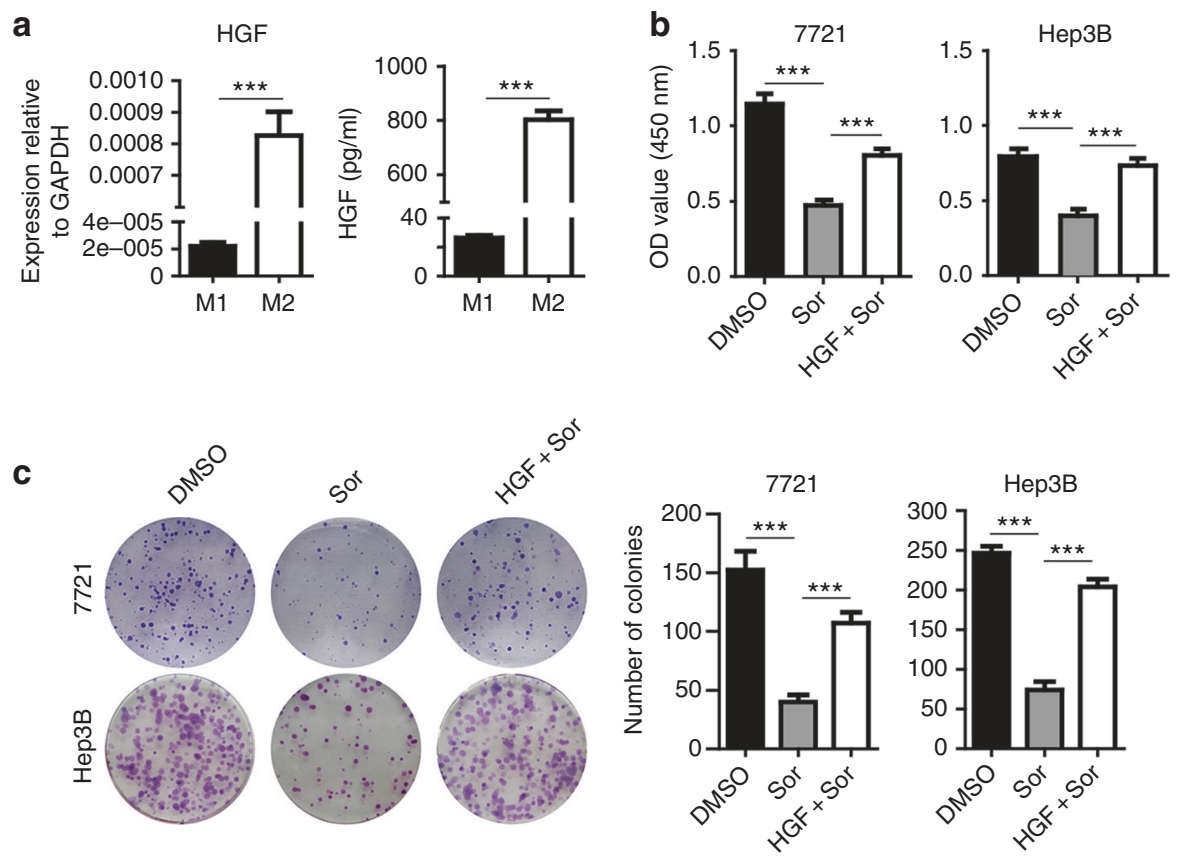

d
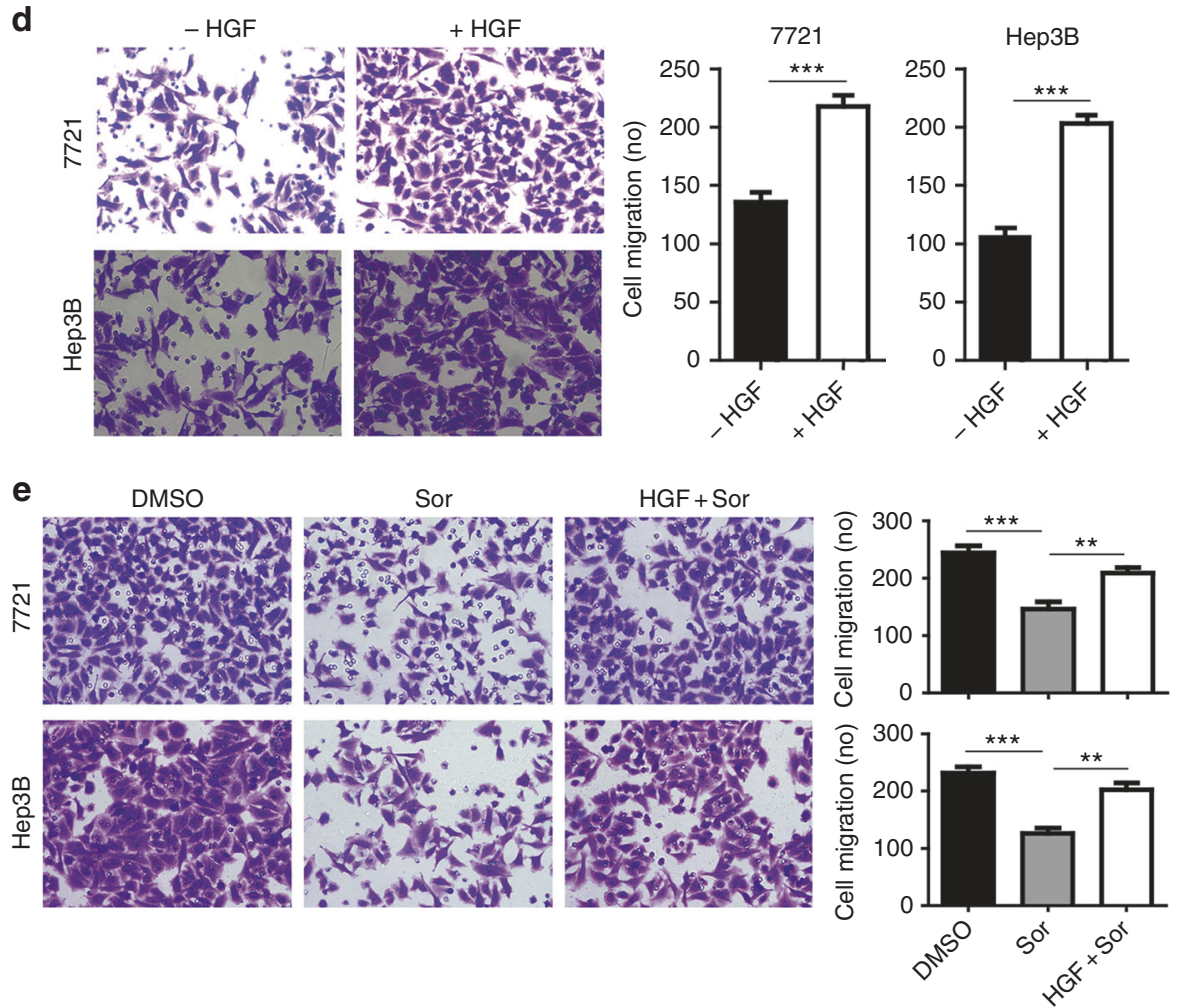

Fig. 3 HGF in CM mediates sorafenib resistance conferred by M2 macrophages. a M2 macrophages expressed and secreted much higher HGF than that of M1 macrophages as determined by RT-qPCR (at mRNA level) and by ELISA assays (protein in CM) after macrophages were cultured in serum-free medium for $48 \mathrm{~h}$. b Recombinant human HGF $(50 \mathrm{ng} / \mathrm{ml})$ reduced the inhibitory efficacy of sorafenib on the proliferation of SMMC-7721 or Hep3B cells as measured by CCK-8 assays. c Colony formation assays showing recombinant HGF impaired sorafenib efficacy on suppression of SMMC-7721 or Hep3B proliferation. d Transwell assays showing recombinant HGF significantly increased the migration of SMMC-7721 or Hep3B cells. e Recombinant HGF impaired the suppressive efficacy of sorafenib on the migration SMMC-7721 or Hep3B cells. $N=3$, mean $\pm \mathrm{SEM},{ }^{* *} P<0.01,{ }^{* * *} P<0.001$

xenografted tumours in DMSO control group (D1 and D5) and in sorafenib treatment group (S4 and S5) (Figs. 6c, S2) were further implanted in mice to generate SS tumour and SR tumour respectively. Daily treatment with sorafenib greatly suppressed the growth of SS tumour and reduced the tumour size by day 5 (SS-Sor versus SS-DMSO, $P<0.01$ ); however, the treatment only marginally inhibited the growth of SR tumour and reduced the tumour size by day 5 (SR-Sor versus SR-DMSO, $P>0.05$ ) (Fig. $6 \mathrm{~d}$ ), 
a

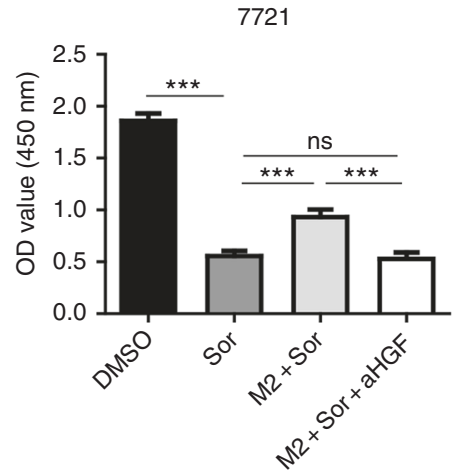

b
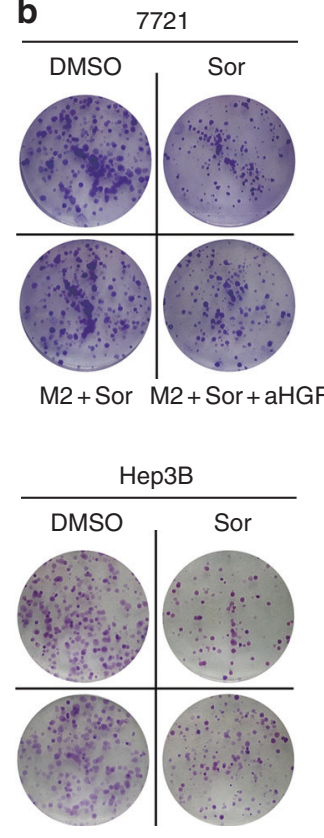

$\mathrm{M} 2+$ Sor $\mathrm{M} 2+$ Sor + aHGF
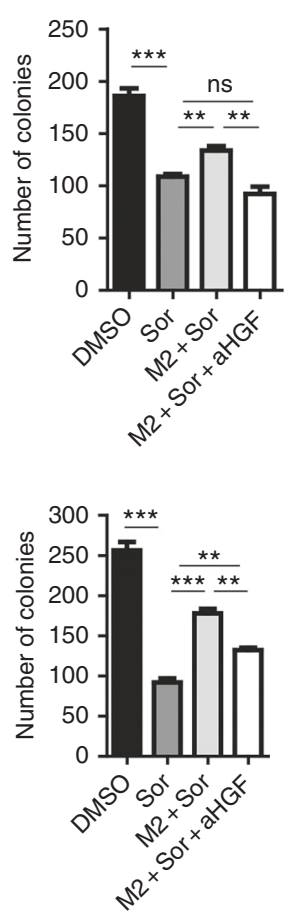

Нер3B

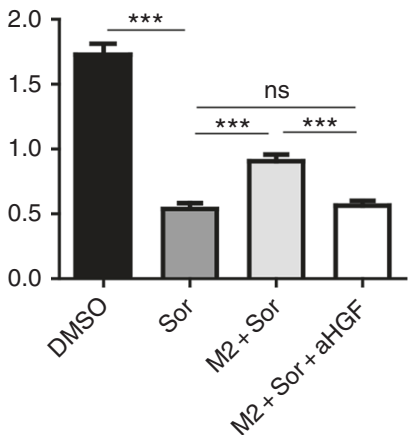

C
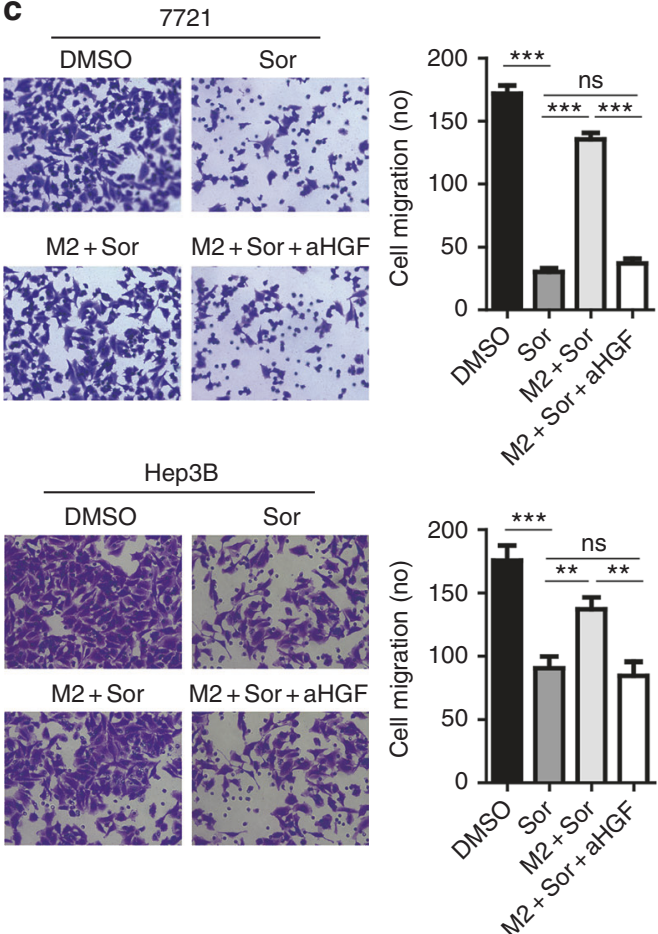

Fig. 4 Anti-HGF antibody abolished the ability of M2-CM to promote the proliferation, colony formation and migration of hepatoma cells. a After incubation of SMMC-7721 and Hep3B cells with $3 \mu \mathrm{M}$ sorafenib in the presence or absence of anti-HGF antibody (aHGF) (200 ng/ml) for $48 \mathrm{~h}$, cell proliferation was examined by CCK-8 assays. b Anti-HGF antibody abolished the effect of M2-CM on sorafenib inhibition of the colony formation of SMMC-7721 and Hep3B cells. c Anti-HGF antibody abolished the effect of M2-CM on the sorafenib inhibition of the migration of SMMC-7721 and Hep3B cells. $N=3$, mean \pm SEM, ${ }^{* *} P<0.01,{ }^{* *} P<0.001$, NS, no significance

confirming the sorafenib resistance. Vascular examination by CD31 staining on xenograft sections did not show significant difference of the endothelial density between SR-DMSO tumour and SS-DMSO tumour; however, more larger vessels were visualised in SR-DMSO tumour than in SS-DMSO tumour and these larger vessels appeared to be less sensitive to sorafenib treatment (Fig. S3). We then investigated the expression of macrophage (F4/80) and M2 macrophage (CD206) markers on cells isolated from xenograft tissues by flow cytometry (Fig. 6e). $\mathrm{F} 4 / 80^{+}$cells isolated from SR-Sor tumour were slightly higher than that of SR-DMSO tumour or SS-DMSO tumour but no statistical difference (SR-Sor versus SR-DMSO or SR-Sor versus SS-DMSO, $P>$ $0.05)$; however, $\mathrm{F} 4 / 80^{+} / \mathrm{CD} 206^{+}$double positive cells isolated from SR-DMSO tumour were significantly higher than that of SR-Sor tumour or SS-DMSO tumour (either SR-DMSO versus SR-Sor or SRDMSO versus SS-DMSO, $P<0.05$ ), highlighting the role of $M 2$-like TAMs in sorafenib resistance.

\section{DISCUSSION}

Macrophages, a major component in $\mathrm{TME}^{38}$ are mainly recruited from the blood circulation monocytes and resided in tumour tissues (TAM) by cytokine CSF-1, chemokines CCL2, CCL9, CCL17, CCL18, and periostin. TAMs have the potential to elicit tumourdestructive reactions (antitumour activity) or drive tumour initiation, angiogenesis, metastasis and suppression of $\mathrm{T}$ cell immunity (pro-tumour activity). ${ }^{39}$ In HCC, the role of TAMs in clinical outcomes remains controversial ${ }^{40-43}$ which may due to the high plasticity and heterogeneity of TAMs. In fact, macrophages are able to differentiate to classically ( $\mathrm{M} 1$, to mirror $\left.\mathrm{T}_{H} 1\right)$ and alternatively ( $M 2$, to mirror $\left.T_{H} 2\right)$ activated macrophages under certain circumstances at the extremes of continuum of functional spectrum. These populations are often distinguished by inducing stimuli (e.g., CSF-1, LPS, and IFN- $\gamma$ for M1; GM-CSF, IL-4 and IL-13 for M2), secretion profiles (e.g., IL-12 ${ }^{\text {high }}, I_{L}-6^{\text {high }}$, TNFa high, and CXCL9 $9^{\text {high }}$ for M1; IL- $10^{\text {high }}$ and TGF- $\beta 1^{\text {high }}$ for M2), protein markers 

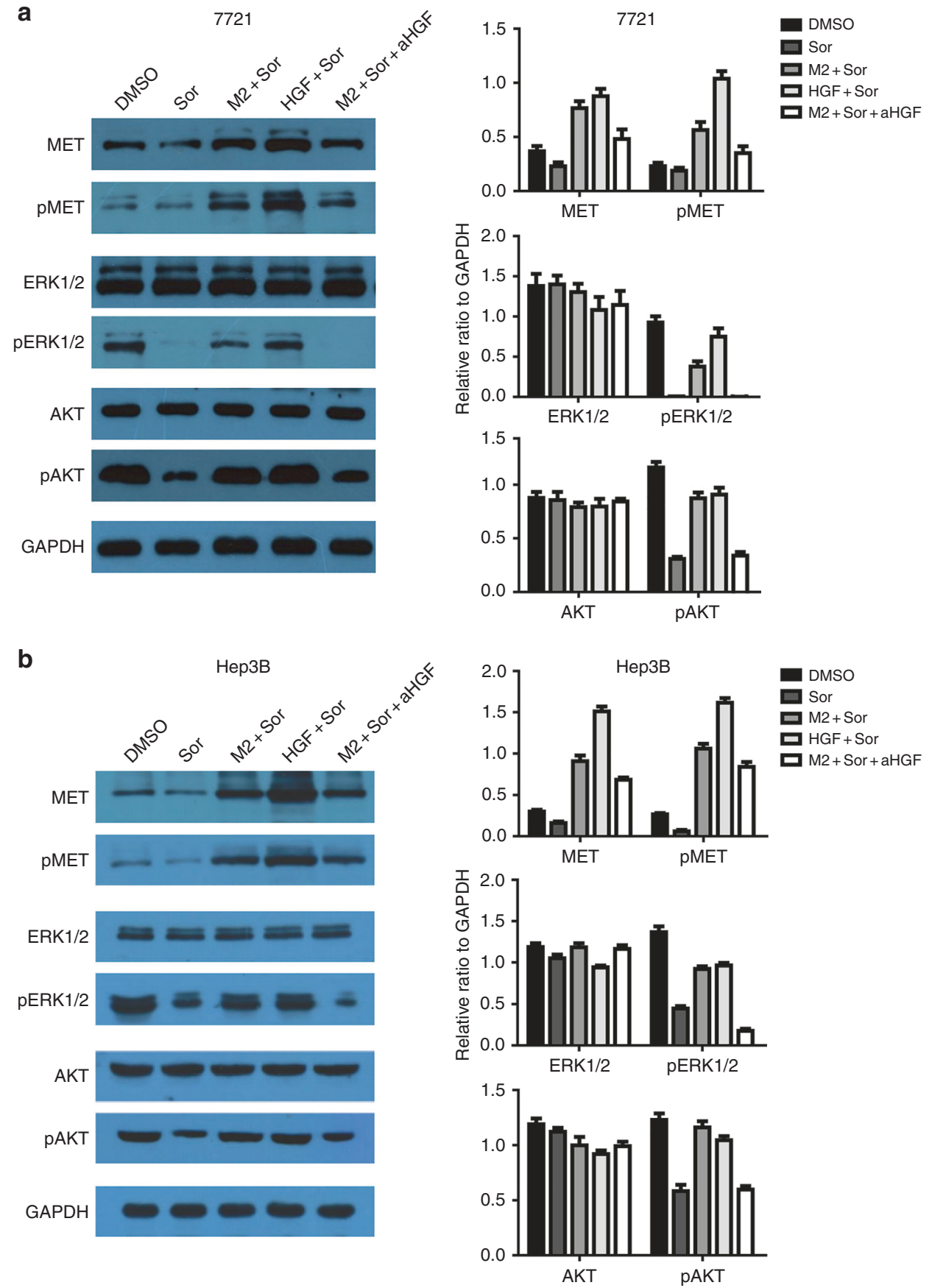

Fig. $5 \mathrm{M} 2$ macrophages confer sorafenib resistance by activation of ERK/MAPK and PI3K/AKT pathways through HGF/c-Met signalling. a Treatment with sorafenib, M2-CM, HGF or anti-HGF antibody (aHGF) alone or combination affects the expression and phosphorylation of c-Met, ERK and AKT kinases in SMMC-7721 cells as measured by Western blots. GAPDH is a protein loading control. b Treatment with sorafenib, M2-CM, HGF or anti-HGF antibody (aHGF) alone or combination affects the expression and phosphorylation of c-Met, ERK, and AKT kinases in Hep3B cells as measured by Western blots. GAPDH is a protein loading control

(e.g., HLA-DR and NOS2 for M1; CD163, CD204/MSR1/SR-A, CD206/ MRC1, CD115/CSF-1R, and Arg1 for M2), and functional characteristics (e.g., antitumour or pro-tumour activity). ${ }^{44-46} \mathrm{CD} 11 \mathrm{~b}$ and CD68 are among the markers commonly used to identify panmonocyte/macrophages.

It has been demonstrated in HCC that M2 macrophages can improve EMT tumour metastasis through production IL-1 $\beta$ and $\mathrm{HGF}^{47}$ while $\mathrm{M} 1$ macrophages inhibits tumour metastasis by distinct integrin-Rho GTPase-Hippo pathways. ${ }^{26}$ Interestingly, sorafenib was recently reported to induce EMT and promote invasiveness and metastasis of HCC cells by downregulation of HTATIP2 expression via JAK-STAT3 signalling in orthotopic mouse models. $^{48}$

We investigated the role of TAMs in sorafenib resistance in HCC. To do this, we first used the human monocytic cell line THP-1 to establish M1 macrophage and M2 macrophage models by differentiating the cells with PMA and consequently polarised the differentiated macrophages with LPS plus IFN- $\gamma$ or IL-4 plus 

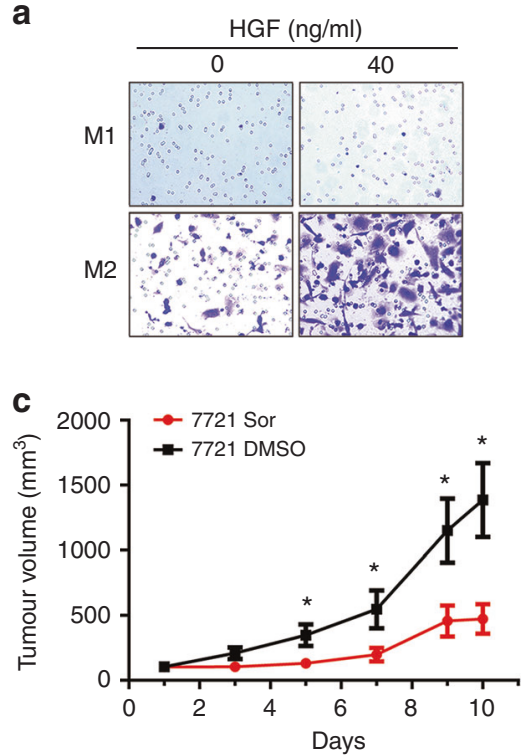

e
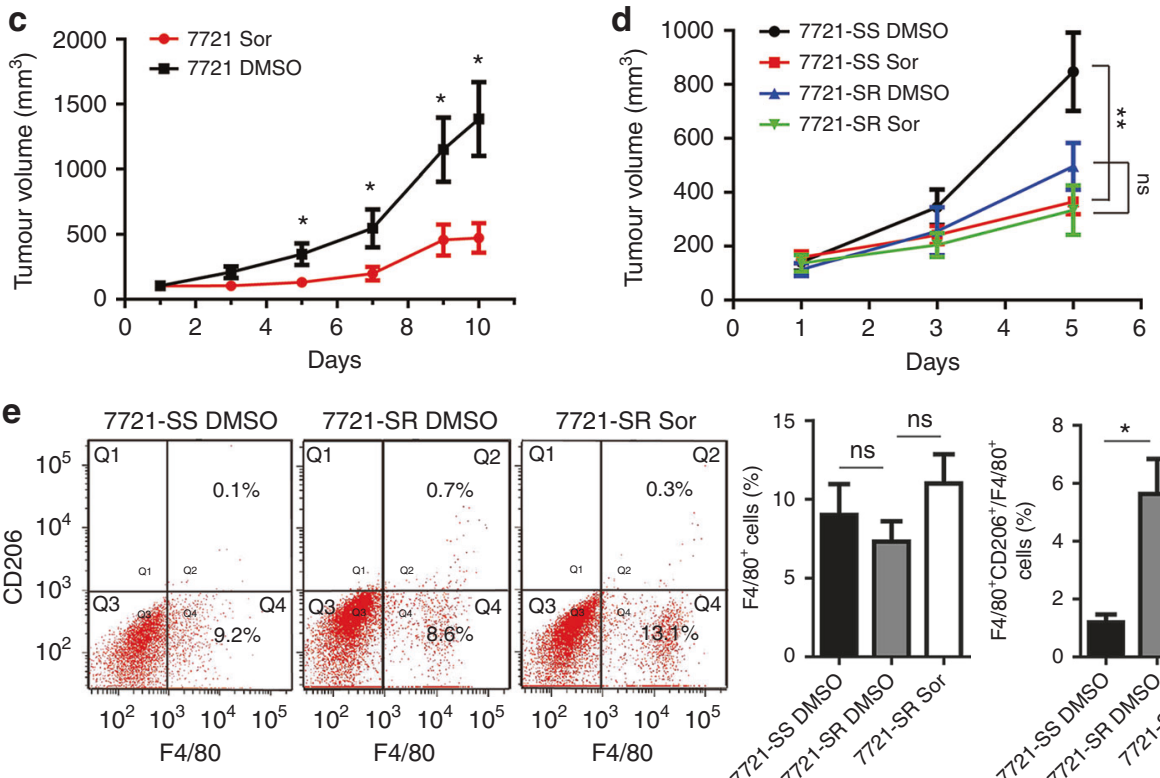

b

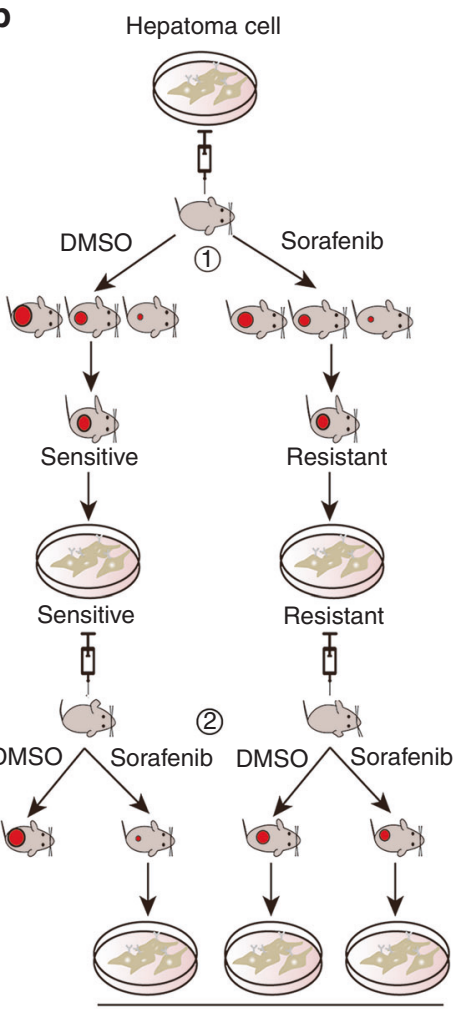

Flow cytometry (M2\%)

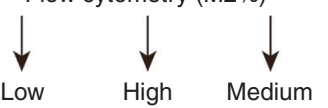

Fig. $6 \mathrm{HGF}$ chemoattracts the migration of tumour-associated M2 macrophages accumulated in sorafenib-resistant tumours. a HGF chemoattracts the migration of $\mathrm{M} 2$ macrophage but not M1 macrophage as determined by transwell migration assays. $\mathbf{b}$ Experimental procedures for development of sorafenib-resistant xenograft models by passage of HCC cells in nude mice, inducible treatment of mice with sorafenib, and implantation of mouse-passaged tumour cells in mice again. c Daily sorafenib treatment inhibited hepatoma growth in immunocompromised mice for the first round of xenograft. $N=5-6$, Mean \pm SEM, ${ }^{*} P<0.05$. Tumour growth in individual mouse was shown in Supplementary Fig. 2a. d Daily sorafenib treatment of the mice greatly inhibited the tumour growth on sorafenib-sensitive (SS) tumour models (SS-Sor versus SS-DMSO, $P<0.01$ ) but marginally affected the tumour growth on sorafenib-resistant (SR) tumour models (SR-Sor versus SR-DMSO, $P>0.05) . N=5-7$, mean $\pm \mathrm{SEM},{ }^{* *} P<0.01$, NS, no significance. e Flow cytometry assays showing SR-DMSO tumour contains more $\mathrm{F} 4 / 80^{+}$and $\mathrm{CD} 206^{+}$double positive $\mathrm{M} 2$ macrophages than that of SR-Sor tumour [SR-DMSO $(N=3)$ versus $\mathrm{SR}-\mathrm{Sor}(N=4), P<0.05$ ] or SS-DMSO tumour [SR-DMSO $(N=3)$ versus SS-DMSO $(N=3), P<0.05)$ ]. Mean $\pm \mathrm{SEM},{ }^{*} P<0.05$, NS, no significance

IL-13 respectively. ${ }^{35,36}$ Identifications of morphological phenotypes, gene expression genotypes and macrophage marker expressions demonstrated that the THP-1-derived $M 1$ and $M 2$ macrophages resemble to tumour-associated M1 and M2 macrophages respectively in multiple aspects. Co-culture of HCC cells (SMMC 7721, Hep3B, and Sk-Hep1) with THP-1 CM showed M1 macrophages did not affect the proliferation and colony formation of tumour cells while M2 macrophages significantly promoted the proliferation and colony formation. Sorafenib potently inhibited the growth, colony formation, and migration of hepatoma cells. Intriguingly, M2-CM significantly attenuated the abilities of sorafenib to inhibit the growth, colony formation and migration of hepatoma cells while M1-CM did not have such effects, suggesting that $M 2$ but not $M 1$ macrophages contribute to sorafenib resistance in HCC by secreting soluble factor(s).

It is known that TAMs produce a variety of factors, ${ }^{38,39}$ some of which such as CCL22 and IGF-1 may contribute to sorafenib resistance. ${ }^{28,29,31,49}$ By screened gene expressions differentially between $M 1$ and $M 2$ macrophages, we found $M 2$ macrophages express HGF abundantly and secret approximately 30 -folds of HGF more than M1 macrophages did. Substitute of M2-CM with recombinant human HGF mitigated the capabilities of sorafenib to inhibit the growth, colony formation and migration of hepatoma cells. Addition of anti-HGF to M2-CM abolished the activities of $\mathrm{M} 2-\mathrm{CM}$ on sorafenib, suggesting that HGF in M2-CM play the predominant role in hepatoma resistance to sorafenib conferred by $\mathrm{M} 2$ macrophages via sustaining tumour growth and metastasis. Apart from tumour-associated M2 macrophages, cancerassociated fibroblasts and hepatoma cells ${ }^{50,51}$ also produce a significant amount of HGF that may contribute to the acquisition of sorafenib resistance. To further explore underlying mechanisms, we assessed the expression and phosphorylation of key proteins in HGF/c-Met, ERK/MAPK and PI3K/AKT pathways in hepatoma cells. We found that sorafenib did not significantly affect protein expressions of c-Met, ERK1/2 and AKT as well as the phosphorylation of pMet, but significantly decreased the phosphorylation of $\mathrm{pERK} / 1 / 2$ and $\mathrm{PAKT}$, confirming the role of sorafenib in inhibition of ERK1/2/MAPK pathway by targeting RAF. ${ }^{10,11}$ Combination of M2-CM or recombinant HGF with sorafenib significantly increased the expression of c-Met and the phosphorylations of pMet, pERK1/ 2 , and PAKT; adding anti-HGF antibody to the combination of M2$\mathrm{CM}$ and sorafenib had the same effects as sorafenib alone. Taken 
together, the results suggest that (a) sorafenib itself does not affect HGF/c-Met signalling but inhibits ERK1/2/MAPK signalling by targeting RAF, and PI3K/AKT signalling by the ERK1/2/MAPK pathway or other mechanism(s); (b) HGF secreted by M2 macrophages activates HGF/c-Met signalling and the downstream ERK1/2/MAPK and PI3K/AKT pathways. ${ }^{52,53}$ Indeed, PI3K has been shown to be involved in sorafenib resistance in $\mathrm{HCC}{ }^{19,20}$

In this study, we show HGF promotes the migration of both hepatoma cells and M2 macrophages, suggesting HGF secreted by tumour-associated M2 macrophages may recruit more macrophages into tumours, regulate the distribution of M2 macrophages in tumour tissues, and thus increase tumour resistance to sorafenib in a feed-forward manner. We further developed xenograft sorafenib-resistant and sorafenib-sensitive models by passage of HCC cells in nude mice, inducible treatment of mice with sorafenib, and implantation of mouse-passaged tumour cells in mice again. ${ }^{16}$ We found $\mathrm{F} 4 / 80^{+} / \mathrm{CD} 206^{+}$double positive $\mathrm{M} 2$ macrophages are significantly higher in SR tumour than that of SS tumour. Short-term sorafenib treatment decreased the amount of F4/80 ${ }^{+} / \mathrm{CD} 206^{+} \mathrm{M} 2$ macrophages (see Fig. 6e) but long-term sorafenib exposure increased HGF synthesis and secretion, along with increased levels of c-Met and pMet. ${ }^{50,51}$ Since sorafenib is also an antiangiogenic agent, the anti-tumour effect might be associated with the antiangiogenic activity, ${ }^{54}$ off-tumour adverse effects $^{55}$ and even tumour metastasis ${ }^{56}$ under certain circumstances. ${ }^{57}$ We examined tumour vasculature in SR and SS tumours by CD31 staining. No significant difference for the endothelial density was observed between SR tumour and SS tumour but more larger vessels existed in SR tumour than in SS tumour, suggesting that such larger vessels may also contribute to sorafenib resistance. ${ }^{37}$

In HCC clinical samples, it is noted that $\mathrm{CD}_{206}{ }^{+} \mathrm{M} 2$ macrophages were highly accumulated in peritumour area, ${ }^{49} \mathrm{a}$ fraction of which highly expressed c-Met. ${ }^{58}$ Increased c-Met expression was independently associated with poor survival in multivariate analysis. ${ }^{59}$ Importantly, a recent biomarker analysis of samples from the pivotal phase III trial of sorafenib showed a trend toward improved survival in patient with a lower pre-treatment plasma HGF concentration, ${ }^{60}$ highlighting the clinical importance of tumour-associated M2 macrophages and HGF in tumour response to sorafenib. Cabozantinib, another multiple kinase inhibitor with most potent inhibition of c-Met, was recently shown to improve median overall survival from 8.0 to 10.2 months as a second line agent in the CELESTIAL phase III trial of HCC. ${ }^{61}$ Taken all together, we postulated combination of sorafenib with cabozantinib will be likely to improve the first line systemic therapeutic efficacy.

In conclusion, we demonstrated that tumour-associated M2 macrophages but not M1 macrophages increase the growth and migration of hepatoma cells, confer hepatoma resistance to sorafenib treatment by secreting HGF. HGF from $\mathrm{M} 2$ macrophages and tumour cells activates HGF/c-Met, MAPK/ERK1/2, and PI3K/ AKT pathways in tumour cells, recruits more macrophages from surrounding blood circulation, regulates the distribution of M2 macrophages in tumour tissues, and increases tumour resistance to sorafenib in a feed-forward manner (Fig. S4). Our results provide new insights into sorafenib resistance in HCC and advocate the development of new trials for the first line systemic therapy by combination of sorafenib with a potent HGF inhibitor such as cabozantinib to improve the first line systemic therapeutic efficacy.

\section{ACKNOWLEDGEMENTS}

We thank Wenbo Hao, Weiwen Xu, Xuexi Yang, Tiancai Liu, Qiang Ma, Hongyan Du, Yao Chen, and Xiaoqing Liao from the School of Laboratory Medicine and Biotechnology, Southern Medical University for their help and support. We thank
Professor Yiguang Lin from School of Life Sciences, University of Technology Sydney, Australia for his constructive editing manuscript.

\section{AUTHOR CONTRIBUTIONS}

N.D., X.S., and J.-L.L. designed research; N.D., X.S., S.W., Y.G., Z.K., Q.X., Y.L., and H.D. performed research; N.D., X.S., S.W., and J.-L.L. analysed data; Y.W., M.L., and J.L.-L. supervised research; N.D. draughted the manuscript; J.-L.L. wrote the paper; all authors approved the manuscript.

\section{ADDITIONAL INFORMATION}

Supplementary information is available for this paper at https://doi.org/10.1038/ s41416-019-0482-x.

Competing interests: The authors declare no competing interests.

Ethics approval and consent to participate: All experimental procedures in animal work were approved by the Ethical Committee of Southern Medical University. Animal welfare was closely monitored in accordance with the Guide for the Care and Use of Laboratory Animals of the National Institutes of Health. This study was performed in accordance with the Declaration of Helsinki.

Funding: This work was financially supported by the National Natural Science Foundation of China (No. 81171959, 81472604, 81201663, and 81874190), Wenzhou Medical University School of Biomedical Engineering and Eye Hospital, and University of Plymouth Faculty of Medicine and Dentistry, UK.

Consent for publication: No consent was involved in this publication.

Data availability: All data and materials generated during and/or analysed during the current study are available from the corresponding author on reasonable request.

Publisher's note: Springer Nature remains neutral with regard to jurisdictional claims in published maps and institutional affiliations.

\section{REFERENCES}

1. Bray, F., Ferlay, J., Soerjomataram, I., Siegel, R. L., Torre, L. A., Jemal, A. Global cancer statistics 2018: GLOBOCAN estimates of incidence and mortality worldwide for 36 cancers in 185 countries. Cancer J. Clin. 68, 394-424 (2018).

2. Forner, A., Reig, M., Bruix, J. Hepatocellular carcinoma. Lancet 391, 1301-1314 (2018).

3. Cheng, A. L., Kang, Y. K., Chen, Z., Tsao, C. J., Qin, S., Kim, J. S. et al. Efficacy and safety of sorafenib in patients in the Asia-Pacific region with advanced hepatocellular carcinoma: a phase III randomised, double-blind, placebo-controlled trial. Lancet Oncol. 10, 25-34 (2009).

4. Kudo, M., Finn, R. S., Qin, S., Han, K. H., Ikeda, K., Piscaglia, F. et al. Lenvatinib versus sorafenib in first-line treatment of patients with unresectable hepatocellular carcinoma: a randomised phase 3 non-inferiority trial. Lancet 391, 1163-1173 (2018).

5. Llovet, J. M., Ricci, S., Mazzaferro, V., Hilgard, P., Gane, E., Blanc, J. F. et al. Sorafenib in advanced hepatocellular carcinoma. N. Engl. J. Med. 359, 378-390 (2008).

6. Bruix, J., Qin, S., Merle, P., Granito, A., Huang, Y. H., Bodoky, G. et al. Regorafenib for patients with hepatocellular carcinoma who progressed on sorafenib treatment (RESORCE): a randomised, double-blind, placebo-controlled, phase 3 trial. Lancet 389, 56-66 (2017).

7. Finn, R. S., Merle, P., Granito, A., Huang, Y. H., Bodoky, G., Pracht, M. et al. Outcomes of sequential treatment with sorafenib followed by regorafenib for HCC: additional analyses from the phase III RESORCE trial. J. Hepatol. 69, 353-358 (2018).

8. El-Khoueiry, A. B., Sangro, B., Yau, T., Crocenzi, T. S., Kudo, M., Hsu, C. et al. Nivolumab in patients with advanced hepatocellular carcinoma (CheckMate 040): an open-label, non-comparative, phase $1 / 2$ dose escalation and expansion trial. Lancet 389, 2492-2502 (2017).

9. Heinrich, B., Czauderna, C., Marquardt, J. U. Immunotherapy of hepatocellular carcinoma. Oncol. Res. Treat. 41, 292-297 (2018).

10. Wilhelm, S., Carter, C., Lynch, M., Lowinger, T., Dumas, J., Smith, R. A. et al. Discovery and development of sorafenib: a multikinase inhibitor for treating cancer. Nat. Rev. Drug Discov. 5, 835-844 (2006).

11. Wilhelm, S. M., Adnane, L., Newell, P., Villanueva, A., Llovet, J. M., Lynch, M. Preclinical overview of sorafenib, a multikinase inhibitor that targets both Raf and 
VEGF and PDGF receptor tyrosine kinase signaling. Mol. Cancer Ther. 7, 3129-3140 (2008)

12. Sia, D., Jiao, Y., Martinez-Quetglas, I., Kuchuk, O., Villacorta-Martin, C., Castro de Moura, M. et al. Identification of an immune-specific class of hepatocellular carcinoma, based on molecular features. Gastroenterology 153, 812-826 (2017).

13. Llovet, J. M., Villanueva, A., Lachenmayer, A., Finn, R. S. Advances in targeted therapies for hepatocellular carcinoma in the genomic era. Nat. Rev. Clin. Oncol. 12, 436 (2015).

14. Berasain, C. Hepatocellular carcinoma and sorafenib: too many resistance mechanisms? Gut 62, 1674-1675 (2013).

15. Huang, X. Y., Ke, A. W., Shi, G. M., Zhang, X., Zhang, C., Shi, Y. H. et al. alphaBcrystallin complexes with 14-3-3zeta to induce epithelial-mesenchymal transition and resistance to sorafenib in hepatocellular carcinoma. Hepatology 57, 2235-2247 (2013).

16. Tovar, V., Cornella, H., Moeini, A., Vidal, S., Hoshida, Y., Sia, D. et al. Tumour initiating cells and IGF/FGF signalling contribute to sorafenib resistance in hepatocellular carcinoma. Gut 66, 530-540 (2017).

17. Xin, H. W., Ambe, C. M., Hari, D. M., Wiegand, G. W., Miller, T. C., Chen, J. Q. et al. Label-retaining liver cancer cells are relatively resistant to sorafenib. Gut $\mathbf{6 2}$, 1777-1786 (2013).

18. Blivet-Van Eggelpoel, M. J., Chettouh, H., Fartoux, L., Aoudjehane, L., Barbu, V., Rey, C. et al. Epidermal growth factor receptor and HER-3 restrict cell response to sorafenib in hepatocellular carcinoma cells. J. Hepatol. 57, 108-115 (2012).

19. Serova, M., de Gramont, A., Tijeras-Raballand, A., Dos Santos, C., Riveiro, M. E., Slimane, K. et al. Benchmarking effects of mTOR, PI3K, and dual PI3K/mTOR inhibitors in hepatocellular and renal cell carcinoma models developing resistance to sunitinib and sorafenib. Cancer Chemother. Pharm. 71, 1297-1307 (2013).

20. Zhang, P. F., Li, K. S., Shen, Y. H., Gao, P. T., Dong, Z. R., Cai, J. B. et al. Galectin-1 induces hepatocellular carcinoma EMT and sorafenib resistance by activating FAK/PI3K/AKT signaling. Cell Death Dis. 7, e2201 (2016).

21. Niu, L., Liu, L., Yang, S., Ren, J., Lai, P. B. S., Chen, G. G. New insights into sorafenib resistance in hepatocellular carcinoma: responsible mechanisms and promising strategies. Biochim Biophys. Acta Rev. Cancer 1868, 564-570 (2017).

22. Liang, Y., Zheng, T., Song, R., Wang, J., Yin, D., Wang, L. et al. Hypoxia-mediated sorafenib resistance can be overcome by EF24 through Von Hippel-Lindau tumor suppressor-dependent HIF-1alpha inhibition in hepatocellular carcinoma. Hepatology 57, 1847-1857 (2013).

23. Shen, Y. C., Ou, D. L., Hsu, C., Lin, K. L., Chang, C. Y., Lin, C. Y. et al. Activating oxidative phosphorylation by a pyruvate dehydrogenase kinase inhibitor overcomes sorafenib resistance of hepatocellular carcinoma. Br. J. Cancer 108, 72-81 (2013).

24. Shimizu, S., Takehara, T., Hikita, H., Kodama, T., Tsunematsu, H., Miyagi, T. et al. Inhibition of autophagy potentiates the antitumor effect of the multikinase inhibitor sorafenib in hepatocellular carcinoma. Int J. Cancer 131, 548-557 (2012).

25. Hernandez-Gea, V., Toffanin, S., Friedman, S. L., Llovet, J. M. Role of the microenvironment in the pathogenesis and treatment of hepatocellular carcinoma. Gastroenterology 144, 512-527 (2013).

26. Zhang, Y. L., Li, Q., Yang, X. M., Fang, F., Li, J., Wang, Y. H. et al. SPON2 promotes M1-like macrophage recruitment and Inhibits hepatocellular carcinoma metastasis by distinct Integrin-Rho GTPase-Hippo pathways. Cancer Res. 78, 2305-2317 (2018).

27. Zhou, S. L., Zhou, Z. J., Hu, Z. Q., Huang, X. W., Wang, Z., Chen, E. B. et al. Tumorassociated neutrophils recruit macrophages and T-regulatory cells to promote progression of hepatocellular carcinoma and resistance to sorafenib. Gastroenterology 150, 1646-1658 (2016).

28. Chen, Y., Huang, Y., Reiberger, T., Duyverman, A. M., Huang, P., Samuel, R. et al. Differential effects of sorafenib on liver versus tumor fibrosis mediated by stromal-derived factor 1 alpha/C-X-C receptor type 4 axis and myeloid differentiation antigen-positive myeloid cell infiltration in mice. Hepatology 59, 1435-1447 (2014).

29. Chen, Y., Ramjiawan, R. R., Reiberger, T., Ng, M. R., Hato, T., Huang, Y. et al. CXCR4 inhibition in tumor microenvironment facilitates anti-programmed death receptor-1 immunotherapy in sorafenib-treated hepatocellular carcinoma in mice. Hepatology 61, 1591-1602 (2015).

30. Sprinzl, M. F., Reisinger, F., Puschnik, A., Ringelhan, M., Ackermann, K., Hartmann, D. et al. Sorafenib perpetuates cellular anticancer effector functions by modulating the crosstalk between macrophages and natural killer cells. Hepatology 57, 2358-2368 (2013)

31. Sprinzl, M. F., Puschnik, A., Schlitter, A. M., Schad, A., Ackermann, K., Esposito, I. et al. Sorafenib inhibits macrophage-induced growth of hepatoma cells by interference with insulin-like growth factor-1 secretion. J. Hepatol. 62, 863-870 (2015).

32. Yang, Y., Ye, Y. C., Chen, Y., Zhao, J. L., Gao, C. C., Han, H. et al. Crosstalk between hepatic tumor cells and macrophages via Wnt/beta-catenin signaling promotes M2-like macrophage polarization and reinforces tumor malignant behaviors. Cell Death Dis. 9, 793 (2018).
33. Zhang, W., Zhu, X. D., Sun, H. C., Xiong, Y. Q., Zhuang, P. Y., Xu, H. X. et al. Depletion of tumor-associated macrophages enhances the effect of sorafenib in metastatic liver cancer models by antimetastatic and antiangiogenic effects. Clin. Cancer Res. 16, 3420-3430 (2010).

34. Wang, S., Huang, X., Li, Y., Lao, H., Zhang, Y., Dong, H. et al. RN181 suppresses hepatocellular carcinoma growth by inhibition of the ERK/MAPK pathway. Hepatology 53, 1932-1942 (2011).

35. Tjiu, J. W., Chen, J. S., Shun, C. T., Lin, S. J., Liao, Y. H., Chu, C. Y. et al. Tumorassociated macrophage-induced invasion and angiogenesis of human basal cell carcinoma cells by cyclooxygenase-2 induction. J. Invest. Dermatol. 129, 1016-1025 (2009).

36. Zhang, F., Liu, H., Jiang, G., Wang, H., Wang, X., Wang, H. et al. Changes in the proteomic profile during the differential polarization status of the human monocyte-derived macrophage THP-1 cell line. Proteomics 15, 773-786 (2015).

37. Li, J. L., Sainson, R. C., Oon, C. E., Turley, H., Leek, R., Sheldon, H. et al. DLL4-Notch signaling mediates tumor resistance to anti-VEGF therapy in vivo. Cancer Res. 71, 6073-6083 (2011).

38. Pollard, J. W. Tumour-educated macrophages promote tumour progression and metastasis. Nat. Rev. Cancer 4, 71-78 (2004).

39. Ruffell, B., Coussens, L. M. Macrophages and therapeutic resistance in cancer. Cancer Cell 27, 462-472 (2015).

40. Li, X., Yao, W., Yuan, Y., Chen, P., Li, B., Li, J. et al. Targeting of tumour-infiltrating macrophages via CCL2/CCR2 signalling as a therapeutic strategy against hepatocellular carcinoma. Gut 66, 157-167 (2017).

41. Li, Y. W., Qiu, S. J., Fan, J., Gao, Q., Zhou, J., Xiao, Y. S. et al. Tumor-infiltrating macrophages can predict favorable prognosis in hepatocellular carcinoma after resection. J. Cancer Res. Clin. Oncol. 135, 439-449 (2009).

42. Waidmann, O., Koberle, V., Bettinger, D., Trojan, J., Zeuzem, S., Schultheiss, M. et al. Diagnostic and prognostic significance of cell death and macrophage activation markers in patients with hepatocellular carcinoma. J. Hepatol. 59, 769-779 (2013).

43. Zhou, J., Ding, T., Pan, W., Zhu, L. Y., Li, L., Zheng, L. Increased intratumoral regulatory $T$ cells are related to intratumoral macrophages and poor prognosis in hepatocellular carcinoma patients. Int. J. Cancer 125, 1640-1648 (2009).

44. Engblom, C., Pfirschke, C., Pittet, M. J. The role of myeloid cells in cancer therapies. Nat. Rev. Cancer 16, 447-462 (2016).

45. Rogers, N. M., Ferenbach, D. A., Isenberg, J. S., Thomson, A. W., Hughes, J. Dendritic cells and macrophages in the kidney: a spectrum of good and evil. Nat. Rev. Nephrol. 10, 625-643 (2014).

46. Zhou, W., Ke, S. Q., Huang, Z., Flavahan, W., Fang, X., Paul, J. et al. Periostin secreted by glioblastoma stem cells recruits $M 2$ tumour-associated macrophages and promotes malignant growth. Nat. Cell Biol. 17, 170-182 (2015).

47. Zhang, J., Zhang, Q., Lou, Y., Fu, Q., Chen, Q., Wei, T. et al. Hypoxia-inducible factor-1alpha/interleukin-1beta signaling enhances hepatoma epithelialmesenchymal transition through macrophages in a hypoxic-inflammatory microenvironment. Hepatology 67, 1872-1889 (2018).

48. Zhang, W., Sun, H. C., Wang, W. Q., Zhang, Q. B., Zhuang, P. Y., Xiong, Y. Q. et al. Sorafenib down-regulates expression of HTATIP2 to promote invasiveness and metastasis of orthotopic hepatocellular carcinoma tumors in mice. Gastroenterology 143, 1641-1649 (2012).

49. Yeung, O. W., Lo, C. M., Ling, C. C., Qi, X., Geng, W., Li, C. X. et al. Alternatively activated (M2) macrophages promote tumour growth and invasiveness in hepatocellular carcinoma. J. Hepatol. 62, 607-616 (2015).

50. Firtina Karagonlar, Z., Koc, D., Iscan, E., Erdal, E., Atabey, N. Elevated hepatocyte growth factor expression as an autocrine c-Met activation mechanism in acquired resistance to sorafenib in hepatocellular carcinoma cells. Cancer Sci. 107, 407-416 (2016).

51. Han, P., Li, H., Jiang, X., Zhai, B., Tan, G., Zhao, D. et al. Dual inhibition of Akt and CMet as a second-line therapy following acquired resistance to sorafenib in hepatocellular carcinoma cells. Mol. Oncol. 11, 320-334 (2017).

52. Goyal, L., Muzumdar, M. D., Zhu, A. X. Targeting the HGF/c-MET pathway in hepatocellular carcinoma. Clin. Cancer Res. 19, 2310-2318 (2013).

53. Hu, C. T., Wu, J. R., Cheng, C. C., Wu, W. S. The therapeutic targeting of HGF/C-Met signaling in hepatocellular carcinoma: alternative approaches. Cancers 9, E58 (2017).

54. Nakamura, M., Zhang, Y., Yang, Y., Sonmez, C., Zheng, W., Huang, G. et al. Offtumor targets compromise antiangiogenic drug sensitivity by inducing kidney erythropoietin production. Proc. Natl Acad. Sci. USA 114, E9635-E9644 (2017).

55. Zhang, Y., Yang, Y., Hosaka, K., Huang, G., Zang, J., Chen, F. et al. Endocrine vasculatures are preferable targets of an antitumor ineffective low dose of antiVEGF therapy. Proc. Natl Acad. Sci. USA 113, 4158-4163 (2016).

56. Yang, Y., Zhang, Y., Iwamoto, H., Hosaka, K., Seki, T., Andersson, P. et al. Discontinuation of anti-VEGF cancer therapy promotes metastasis through a liver revascularization mechanism. Nat. Commun. 7, 12680 (2016). 
57. Zhang, Y., Sun, M., Huang, G., Yin, L., Lai, Q., Yang, Y. et al. Maintenance of antiangiogenic and antitumor effects by orally active low-dose capecitabine for long-term cancer therapy. Proc. Natl Acad. Sci. USA 114, E5226-E5235 (2017).

58. Zhao, L., Wu, Y., Xie, X. D., Chu, Y. F., Li, J. Q., Zheng, L. c-Met identifies a population of matrix metalloproteinase 9-producing monocytes in peritumoural stroma of hepatocellular carcinoma. J. Pathol. 237, 319-329 (2015).

59. Wang, Z. L., Liang, P., Dong, B. W., Yu, X. L., Yu, D. J. Prognostic factors and recurrence of small hepatocellular carcinoma after hepatic resection or microwave ablation: a retrospective study. J. Gastrointest. Surg. 12, 327-337 (2008).

60. Llovet, J. M., Pena, C. E., Lathia, C. D., Shan, M., Meinhardt, G., Bruix, J., Group, S. I. S. Plasma biomarkers as predictors of outcome in patients with advanced hepatocellular carcinoma. Clin. Cancer Res. 18, 2290-2300 (2012).

61. Abou-Alfa, G. K., Meyer, T., Cheng, A. L., El-Khoueiry, A. B., Rimassa, L., Ryoo, B. Y. et al. Cabozantinib in patients with advanced and progressing hepatocellular carcinoma. N. Engl. J. Med. 379, 54-63 (2018).
Open Access This article is licensed under a Creative Commons Attribution 4.0 International License, which permits use, sharing, adaptation, distribution and reproduction in any medium or format, as long as you give appropriate credit to the original author(s) and the source, provide a link to the Creative Commons license, and indicate if changes were made. The images or other third party material in this article are included in the article's Creative Commons license, unless indicated otherwise in a credit line to the material. If material is not included in the article's Creative Commons license and your intended use is not permitted by statutory regulation or exceeds the permitted use, you will need to obtain permission directly from the copyright holder. To view a copy of this license, visit http://creativecommons. org/licenses/by/4.0/.

(c) The Author(s) 2019 